\title{
Effects of Denture Removal on Expiratory Upper Airway Patency during Mask Ventilation in Complete Denture Wearers under General Anesthesia
}

\author{
Takashi Imaizumi, Toshiyuki Yano*, Chiho Uneda, Kazuhiko Kawamoto, Ryosuke Nakayama \\ Division of Anesthesia, Kumamoto Kinoh Hospital, Kumamoto, Japan \\ Email: `yano@juryo.or.jp
}

How to cite this paper: Imaizumi, T., Yano, T., Uneda, C., Kawamoto, K. and Nakayama, R. (2021) Effects of Denture Removal on Expiratory Upper Airway Patency during Mask Ventilation in Complete Denture Wearers under General Anesthesia. Open Journal of Anesthesiology, 11, 39-48.

https://doi.org/10.4236/ojanes.2021.112005

Received: January 6, 2021

Accepted: February 19, 2021

Published: February 22, 2021

Copyright $\odot 2021$ by author(s) and Scientific Research Publishing Inc. This work is licensed under the Creative Commons Attribution International License (CC BY 4.0).

http://creativecommons.org/licenses/by/4.0/

\begin{abstract}
Background: Some anesthetized patients present with expiratory airway obstruction (EAO) during mask ventilation (MV). EAO may occur more frequently among edentulous patients for whom a firmer grip of the mask and the mandible is needed to avoid gas leakage. We compared EAO grades before and after denture removal and identified predictors of EAO during MV in edentulous patients. Methods: One hundred and eight denture-wearing edentulous patients were anesthetized, their mouths were wrapped with film, their nasal masks and mandibles were held with two hands, and their lungs were ventilated in a pressure-controlled mode. Their dentures were subsequently removed, mouths were re-wrapped, and nasal MV was reapplied. EAO was graded based on capnography waveforms as follows: Grade I, normal waveform (no obstruction); II, loss of the alveolar plateau of the waveform (partial obstruction); and III, no waveform (total obstruction). EAO grades were compared before and after denture removal. Predictive demographic variables for occurrence of total EAO were identified using a multivariate analysis. Results: The proportions of Grades I, II, and III were 43.5\%, $26.9 \%$, and $29.6 \%$ when dentures were worn and $35.2 \%, 15.7 \%$, and $49.1 \%$, respectively, when dentures were removed. A significant difference was observed in EAO grades before and after denture removal $(P=0.000034)$. Age $\geq$ 75 years (adjusted odds ratio 3.41; 95\% confidence interval 1.31 - 8.87; $P=$ 0.012 ) and body mass index $\geq 25 \mathrm{~kg} / \mathrm{m}^{2}$ (adjusted odds ratio $2.61 ; 95 \%$ confidence interval $1.07-6.40 ; P=0.036$ ) were independently associated with an increased incidence of total EAO in patients with their dentures removed. Conclusion: Denture removal impaired expiratory upper airway patency in edentulous patients, particularly in older or obese patients, during MV.
\end{abstract}




\section{Keywords}

Airway Obstruction, Edentulous Jaw, Expiration, Nasopharynx, Soft Palate

\section{Introduction}

Mask ventilation (MV) is a pivotal procedure used to deliver oxygen during the induction of general anesthesia before a secure airway with a tracheal tube or supraglottic airway device can be established. Difficult mask ventilation (DMV) may cause hypoxic tissue injury, resulting in morbidity and mortality related to anesthesia. A lack of teeth is an independent predictor of DMV [1]. In edentulous patients, the seal between the mask and the face is likely to be inadequate due to the loss of structural support from the face, resulting in a gas leak during positive pressure inspiration. To achieve adequate MV in edentulous patients, leaving the dentures in position has been reported to improve the seal between the mask and the face [2].

In anesthetized patients, upper airway obstruction is typically released by a head-tilt and jaw-thrust maneuver during MV. However, the velopharynx may be occluded during expiration [3]. In this case, when the mask is tightly seated onto the face and the mouth is closed, the soft palate functions as a one-way check valve during expiration, leading to expiratory airway obstruction (EAO). It has recently been demonstrated that EAO concomitant with retropalatal occlusion occurs during MV in dentulous patients [4]. EAO presents as fluttering lips or successive chest rises without a fall due to positive pressure inspiration, which is referred to as "breath stacking" [5]. DMV due to breath stacking may be misdiagnosed as inspiratory airway obstruction [5]. In edentulous patients, the effort needed to hold the mask down over the face with the thumb and index finger and concomitantly grasp the mandible with the other three fingers (EC-clamp technique) tightly to establish a mask seal may shut the mouth and press the tongue backward, thereby promoting the check valve function of the soft palate. We hypothesized that wearing complete dentures retains expiratory nasopharyngeal patency during MV in edentulous patients. In the present study, we primarily tested this hypothesis by comparing nasal capnography waveforms before and after denture removal during MV at the induction of general anesthesia. We also attempted to identify the predictive demographic variables for EAO in patients with their dentures removed.

\section{Materials and Methods}

\subsection{Patients}

This study was conducted at the Surgical Center, Kumamoto Kinoh Hospital, Kumamoto, Japan, between October 2012 and December 2014. The study protocol was approved by the Institutional Ethical Review Committee and registered with the UMIN Clinical Trials Registry (UMIN000011017). Consecutive 
edentulous patients who typically wear complete dentures and were scheduled to undergo elective orthopedic or plastic surgery under general anesthesia were enrolled. They were classified into American Society of Anesthesiologists physical status classes 1 to 3 and did not have a beard, head or neck abnormalities, nasal obstruction, history of palatopharyngeal surgery, abnormal respiratory function, or a known difficult airway. Written informed consent was obtained from all participating patients.

\subsection{Methodology}

Non-premedicated patients with their complete dentures in position without denture adhesives were placed in a supine position with the external ear canal at the same level with the top of the shoulder, and they underwent routine anesthesia monitoring, comprising pulse oximetry, electrocardiography, and non-invasive blood pressure measurements. A standard breathing circuit and 3-L bag were used. The side-stream sampling tubing of end-tidal $\mathrm{CO}_{2}$ device was connected to the gas sampling port of a heat and moisture exchanger $\left(D R^{\mathrm{TM}}\right.$ Hygrobac $\mathrm{S}$, Covidien, Mansfield, USA), which was located between the Y-piece and elbow connector of a face mask. The patient's chest and capnography waveforms were recorded separately using two camcorders throughout the study for a later assessment of breath stacking and EAO grade. The first author (TI), an anesthesiologist with 20 years of experience, was in charge of all anesthesia procedures and interventions. Before induction of anesthesia, a fresh $\mathrm{O}_{2}$ flow was set at 5 $\mathrm{L} / \mathrm{min}$ on the anesthesia machine. After 5 min of preoxygenation using an anatomically shaped adult silicone face mask (Sil-Flex ${ }^{\mathrm{TM}}$ Silicone Mask \# 4, Galemed, I-Lan, Taiwan), patients received remifentanil $0.2 \mu \mathrm{g} / \mathrm{kg}$ and propofol 2 $3 \mathrm{mg} / \mathrm{kg}$ boluses followed by a continuous infusion of remifentanil $(0.2-0.3$ $\mu \mathrm{g} / \mathrm{kg} / \mathrm{min})$ and propofol $(6 \mathrm{mg} / \mathrm{kg} / \mathrm{h})$. Muscle relaxants were not used. After confirming unconsciousness, loss of the eyelash reflex, and respiratory arrest, the first author tilted the head and held both sides of the mask and the mandible with both hands. Jaw thrust was not applied to prevent the dentures from dislodging. Mechanical ventilation was initiated using an anesthesia machine ventilator in the pressure-controlled ventilation (PCV) mode with an inspiratory pressure of $20 \mathrm{hPa}$, respiratory rate of 10 breaths/min, and inspiratory to expiratory ratio of $1: 2$. The inclusion criteria were as follows: 1 ) normal capnography waveforms and 2) an end-tidal $\mathrm{CO}_{2}$ value higher than $30 \mathrm{mmHg}$.

The mouths of patients who met the inclusion criteria were closed and made airtight with a polyurethane transparent adhesive film (Tegaderm ${ }^{\mathrm{TM}}$ Film, $3 \mathrm{M}$ Health Care, St. Paul, MN, USA). The tip of the sampling tubing of end-tidal $\mathrm{CO}_{2}$ device was displaced to the right nasal vestibule, the head was tilted, the nose was entirely covered with a silicone round-shaped face mask made for a child (Sil-Flex ${ }^{\mathrm{TM}}$ Silicone Mask \# 2, Galemed, I-Lan, Taiwan), both sides of the mask and the mandible were held with two hands, and mechanical ventilation via the nasal passage alone was applied in the PCV mode under the same venti- 
lation conditions as described above. At this point, if any sound or vibration was detected between the mask and the face due to a gas leak, the patient was excluded. After recording stable capnography waveforms during at least three respiratory cycles, the film was stripped off, and the dentures were removed. The mouth was subsequently covered with another film, and holding of the mask and ventilation were performed in the same manner. After the capnography waveforms were recorded, the film was stripped off and the trachea was intubated using rocuronium or a supraglottic airway device was placed.

The primary outcome was the grade of EAO based on the patterns of capnography waveforms. The capnography waveforms recorded were classified as follows: Grade I, normal waveform; II, loss of the normal upsloping alveolar plateau (phase III) of the waveform; and III, no waveform. Grades II and III were defined as partial and total EAO, respectively. Video clips of chest movements were analyzed, and cumulative expansion of the chest by successive tidal breaths was defined as breath stacking.

\subsection{Statistical Analyses}

\subsubsection{Sample Size Calculation}

In our preliminary test of fifteen edentulous patients, total EAO occurred in four when wearing their dentures and in seven when their dentures were removed. The calculated sample size to detect this difference by a chi-square test for a type I error of 0.05 and a power of 0.8 was at least 101. In the multivariate logistic regression analysis, at least ten outcomes are needed for each included independent variable according to standard methods. The expected rate of total EAO in patients with their dentures removed (47\%; 7 out of 15) allowed use of 4 variables for 101 samples in a logistic regression model without overfitting.

\subsubsection{Data Analyses}

Data are presented as absolute or mean $\pm \mathrm{SD}$ values. $P$ values $<0.05$ were considered significant. All analyses were performed with the free statistical software EZR version 1.28

(http://www.jichi.ac.jp/saitama-sct/SaitamaHP.files/statmed.html) [6]. The proportions of EAO grades were compared before and after denture removal using a Wilcoxon signed-rank test. To identify independent predictors of total EAO (Grade III) in patients with their dentures removed, a forced entry multivariate logistic regression analysis was conducted with total EAO as a dependent variable. Independent variables included four demographic variables (age, sex, body mass index [BMI], and the Mallampati classification). Adjusted odds ratios (ORs) were reported together with $95 \%$ confidence intervals (CIs).

\section{Results}

One hundred and fifteen patients were enrolled in the present study. Five patients who showed inspiratory airway obstruction and required an oral airway device during the initial MV with two-handed mask holding with the head tilt 
when wearing dentures were excluded for not fulfilling the inclusion criteria. After the study, two patients whose experimental records had some missing data were also excluded. Therefore, 108 patients were included in the study.

Patient demographics are shown in Table 1. Eighty-eight men and 20 women were included, with men accounting for $81.5 \%$ of the participants. The mean age of the patients was 77 years, and $88(81.5 \%)$ patients were 65 years or older. The mean BMI was $25 \mathrm{~kg} / \mathrm{m}^{2}$, and $42(38.9 \%)$ and $10(9.3 \%)$ patients were pre-obese $\left(25 \leq \mathrm{BMI}<30 \mathrm{~kg} / \mathrm{m}^{2}\right)$ and obese $\left(\mathrm{BMI} \geq 30 \mathrm{~kg} / \mathrm{m}^{2}\right)$, respectively. The Mallampati score was not assessed in six patients, and $51(50.0 \%)$ were classified as III or IV.

Differences in EAO grades before and after denture removal are shown in Figure 1(a). Before denture removal, 47 (43.5\%), 29 (26.9\%), and 32 (29.6\%) patients were classified as Grades I, II, and III, respectively. After denture removal, 38 (35.2\%) and 17 (15.7\%) patients were Grades I and II, respectively, while 53 (49.1\%) were Grade III. A significant difference was observed in the proportion of EAO grades before and after denture removal $(P=0.000034)$. Figure $1(\mathrm{~b})$ shows individual changes in EAO grades when the dentures were removed. The EAO grades of 34 out of 47 (72.3\%), 5 out of 29 (17.2\%), and 29 out of 32 (90.6\%) patients classified as Grades I, II, and III, respectively, remained unchanged before and after denture removal. Thirteen out of 47 (27.7\%) and 20 out of $29(69.0 \%)$ patients classified as Grades I and II, respectively, worsened to Grade II or III. However, 4 out of $29(13.8 \%)$ and 3 out of $32(9.4 \%)$ patients classified as Grades II and III, respectively, improved to Grades I and II.

A review of the video clips of chest movements was used to estimate the incidence of breath stacking by visual evaluations. All patients classified as Grade III (total obstruction) showed breath stacking, whereas none of those classified as Grade I (no obstruction) did. In patients classified as Grade II (partial obstruction), 12 out of 29 (41.4\%) before denture removal and 5 out of 17 (29.4\%) after denture removal showed breath stacking.

Multivariate logistic regression analysis by the forced entry of demographic variables, including age, sex, BMI, and the Mallampati classification, revealed that age $\geq 75$ years (OR 3.41; 95\% CI $1.31-8.87 ; P=0.012$ ) and BMI $\geq 25 \mathrm{~kg} / \mathrm{m}^{2}$ (OR 2.61; 95\% CI $1.07-6.40 ; P=0.036$ ) were independently associated with an

Table 1. Demographics of patients.

\begin{tabular}{cc}
\hline Variables & Results \\
\hline Age, years & $77 \pm 8$ \\
Sex (male/female) & $88 / 20$ \\
Weight, $\mathrm{kg}$ & $56 \pm 12$ \\
Height, $\mathrm{cm}$ & $150 \pm 9$ \\
Body mass index, kg/m ${ }^{2}$ & $25 \pm 4$ \\
Mallampati classification (I/II/III/IV/not assessed) & $20 / 31 / 50 / 1 / 6$
\end{tabular}

Total number of patients: 108 . Data are expressed as absolute or mean \pm SD values. 

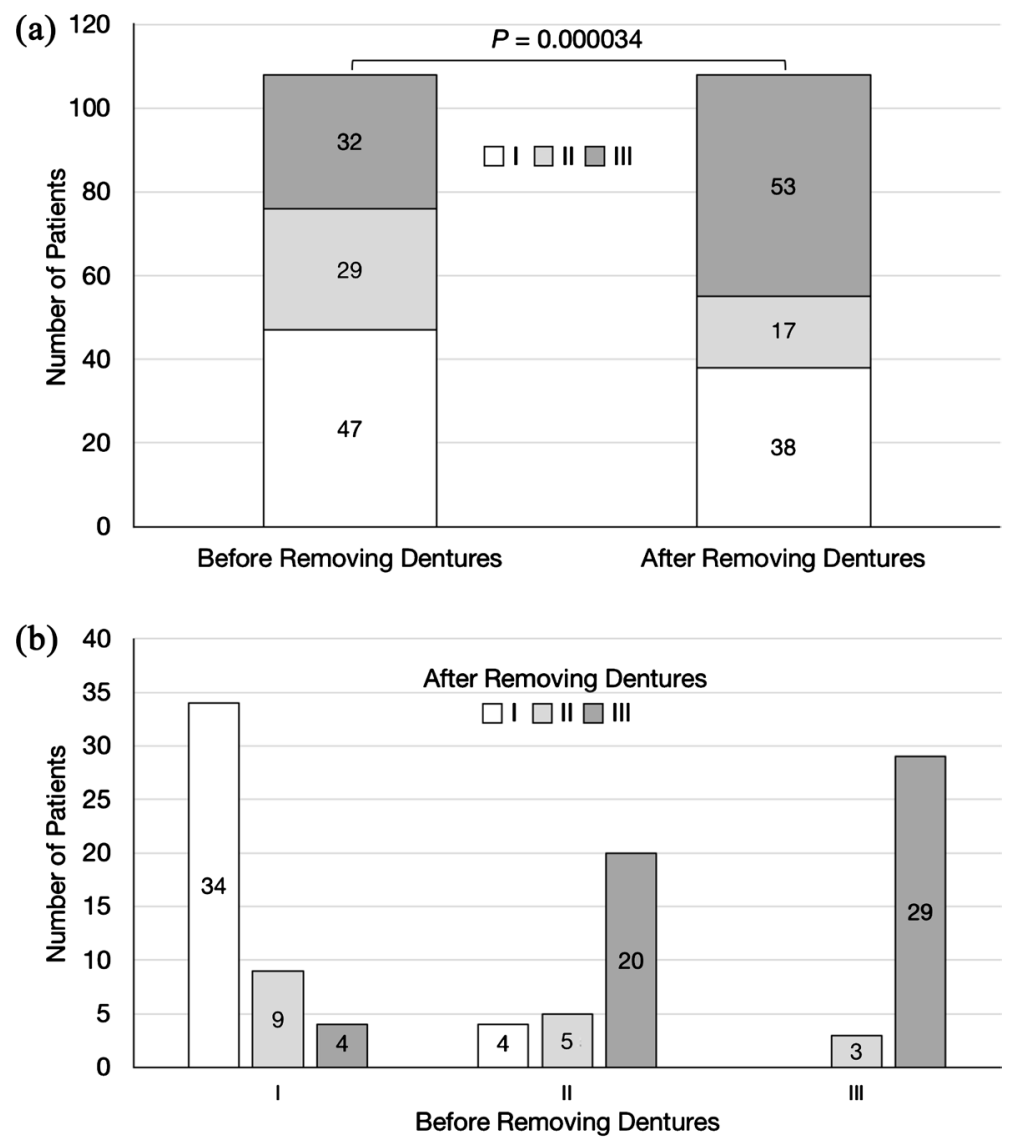

Figure 1. (a) Differences in expiratory airway obstruction (EAO) grading based on capnography waveforms before and after denture removal. (b) Individual changes in EAO grading after dentures were removed. Grade I, no obstruction; II, partial obstruction; III, total obstruction.

Table 2. Predictive demographic variables for total expiratory upper airway obstruction in patients with their dentures removed based on a multivariate logistic regression analysis.

\begin{tabular}{cccc}
\hline Independent variables & Adjusted odds ratio & 95\% confidence interval & $P$ value \\
\hline Age $\geq 75$ years & 3.41 & $1.31-8.87$ & 0.012 \\
Male sex & 2.21 & $0.71-6.94$ & 0.173 \\
Body mass index $\geq 25 \mathrm{~kg} / \mathrm{m}^{2}$ & 2.61 & $1.07-6.40$ & 0.036 \\
Mallampati classification III or IV & 0.66 & $0.28-1.58$ & 0.349 \\
\hline
\end{tabular}

Total number of patients: 102. Data from six patients whose Mallampati classification was not assessed were excluded from this analysis.

increased incidence of total EAO in patients with their dentures removed (Table 2). In this analysis, six patients without Mallampati classifications were excluded.

\section{Discussion}

Herein, we demonstrated that edentulous patients with their dentures removed had a higher incidence of total EAO, qualitatively assessed by capnography 
waveforms and the occurrence of breath stacking, than that seen in the patients before denture removal during MV at the induction of anesthesia. We also identified age $\geq 75$ years and $\mathrm{BMI} \geq 25 \mathrm{~kg} / \mathrm{m}^{2}$ as independent predictors of total EAO in edentulous patients with their dentures removed.

We used three patterns of capnography waveforms (a normal waveform, loss of phase III of the waveform, and no waveform) to grade EAO. These patterns were previously shown to correspond to the ranges of expiratory flow limitation based on expiratory tidal volume and end-tidal $\mathrm{CO}_{2}$ during $\mathrm{MV}$ [7]. We also covered patients' mouths with film to prevent expiration from the mouth for assessment of expiratory nasopharyngeal patency.

Six decades ago, Safer and Redding suggested that EAO was caused by the valve-like behavior of the soft palate during mouth-to-nose artificial breathing [8] [9]. Recently, direct retropalatal closure with the soft palate during the expiratory phase of $\mathrm{MV}$ in patients with and without obstructive sleep apnea has been reported using real-time endoscopy [4]. A textbook of airway management documented occurrence of expiratory nasopharyngeal obstruction by the soft palate in approximately $20 \%$ of patients during MV [3]. In anesthetized, paralyzed patients, including upper and/or lower edentulous individuals, the incidence of total EAO during MV was 34\% [5]. In the present study, the incidence of total EAO before denture removal in unparalyzed patients was 30\%, which was similar to the incidence in the previous study. Our new findings show that removing dentures increased incidence of total EAO by $65 \%$, indicating the deterioration of nasopharyngeal patency.

Wearing dentures has been shown to improve the efficiency of MV in edentulous patients [2]. Edentulism negatively affects the shape of facial soft tissues, which impedes the fitting of the mask to the face, thereby causing a gas leak. Furthermore, cephalad elevation and relative enlargement of the tongue in the oral cavity due to the resorption of the alveolar bone reduce the oropharyngeal space [2] [10]; a tighter EC-clamp of the mask and the mandible for an adequate mask seal may further compromise expiratory upper airway patency in edentulous patients. The wearing of dentures restores the maxillomandibular vertical dimension, the distance between the nose and chin [11], which may increase the oropharyngeal space during MV. Improvements in the structure of the face and oral cavity by wearing dentures may improve expiratory upper airway patency together with better mask holding, thereby contributing to a reduction in the likelihood of DMV in edentulous patients [2]. However, we should note that $30 \%$ of edentulous patients continue to experience EAO even if an adequate mask seal is achieved by wearing dentures during MV, as shown in the present study.

$\mathrm{MV}$ with dentures left in place during anesthesia induction is a measure to reduce occurrence of EAO in edentulous patients; however, there is a risk that dentures will come off and be trapped in the pharynx or larynx. If the retropalatal space is obstructed by the soft palate during expiration, the oral passage will be crucial to prevent breath stacking. The intermittent release of the EC-clamp 
allows stemmed gas from the lungs to escape through the mouth and may reduce the risk of breath stacking. The "triple airway maneuver," which involves mouth opening in addition to a head tilt and jaw thrust by both hands of one person, and the "lower lip face mask placement" with two hands, which consists of placing the caudal end of the face mask just above the inferior lip that automatically opens the mouth, are believed to ameliorate EAO [3] [12]. An oropharyngeal airway can also be used to avoid EAO during one-hand MV. The triple airway maneuver, lower lip face mask placement, and the insertion of the oropharyngeal airway can secure the expiratory oral passage and tentatively increase the oropharyngeal space during MV in edentulous patients.

Macroglossia, a large uvula, and old age were identified as independent predictors of EAO from 33 demographic variables and measures from the face, neck, and airway by a multivariate regression analysis [5]. Edentulous patients frequently have relative macroglossia owing to a reduced space in the oral cavity due to alveolar bone atrophy [10]. The present study showed that increased age and BMI were independent predictors of total $\mathrm{EAO}$ in patients with their dentures removed. The lack of teeth, macroglossia, and increased age and BMI are independent predictors of DMV [1] [13] [14] [15]. Therefore, DMV may be more accurately predicted in older or obese edentulous patients who are susceptible to EAO.

There are several limitations to the present study. In this one-group pretest-posttest study, the observer was not blinded to the presence of dentures, and the order of intervention was not randomized. Furthermore, the stability of dentures, which may affect EAO grading, was unclear. In addition, muscle relaxants were not used during MV. It remains unclear how muscle relaxants may influence the nasal passage in edentulous patients. They do not reduce nasal or oral airflow during MV in anesthetized patients with normal upper airways [16]. Another limitation is that the protocol of anesthesia was similar in all patients; however, the level of anesthesia was not clear because the hypnotic level was not assessed by electroencephalogram-based monitoring. Moreover, the duration of edentulism was not included in the analyses because a large number of participants did not clearly remember the date they received their dentures. The amount of residual bone related to the fitting of dentures depends on the age at which patients became edentulous [2].

\section{Conclusion}

In summary, EAO occurred more frequently in edentulous patients after denture removal than that seen before denture removal, and age $\geq 75$ years and BMI $\geq 25$ $\mathrm{kg} / \mathrm{m}^{2}$ were independent predictors of total EAO during MV in patients with their dentures removed. These results suggest that removing dentures impairs expiratory airway patency in edentulous patients, particularly older or obese patients. Not only a poor mask seal but also EAO may contribute to DMV in edentulous patients with their dentures removed. A quantitative assessment of respi- 
ratory parameters and real-time endoscopy of the velopharynx during MV are needed to elucidate the precise underlying mechanisms of EAO and its relationship with DMV in edentulous patients.

\section{Acknowledgements}

We thank Yuko Takase, the institutional clinical research coordinator, for her generous assistance in obtaining informed consent from the participating patients. This study was supported solely by our departmental funds.

\section{Conflicts of Interest}

The authors declare no conflicts of interest regarding the publication of this paper.

\section{References}

[1] Langeron, O., Masso, E., Huraux, C., Guggiari, M., Bianchi, A., Coriat, P. and Riou, B. (2000) Prediction of Difficult Mask Ventilation. Anesthesiology, 92, 1229-1236. https://doi.org/10.1097/00000542-200005000-00009

[2] Conlon, N.P., Sullivan, R.P., Herbison, P.G., Zacharias, M. and Buggy, D.J. (2007) The Effect of Leaving Dentures in Place on Bag-Mask Ventilation at Induction of General Anesthesia. Anesthesia and Analgesia, 105, 370-373. https://doi.org/10.1213/01.ane.0000267257.45752.31

[3] McGee II, J.A. and Vender, J.S. (2007) Nonintubation Management of the Airway: Mask Ventilation. In: Hagberg, C.A., Ed., Benumof s Airway Management: Principles and Practice, 2nd Edition, Mosby, Philadelphia, 345-370.

[4] Okuyama, M., Kato, S., Sato, S., Okazaki, J., Kitamura, Y., Ishikawa, T., Sato, Y. and Isono, S. (2018) Dynamic Behaviour of the Soft Palate during Nasal Positive Pressure Ventilation under Anaesthesia and Paralysis: Comparison between Patients with and without Obstructive Sleep-Disordered Breathing. British Journal of Anaesthesia, 120, 181-187. https://doi.org/10.1016/j.bja.2017.11.016

[5] Buffington, C.W., Wells, C.M.Q. and Soose, R.J. (2012) Expiratory Upper Airway Obstruction Caused by the Soft Palate during Bag-Mask Ventilation. Open Journal of Anesthesiology, 2, 38-43. https://doi.org/10.4236/ojanes.2012.22010

[6] Kanda, Y. (2013) Investigation of the Freely Available Easy-to-Use Software EZR for Medical Statistics. Bone Marrow Transplantation, 48, 452-458. https://doi.org/10.1038/bmt.2012.244

[7] Sato, S., Hasegawa, M., Okuyama, M., Okazaki, J., Kitamura, Y., Sato, Y., Ishikawa, T., Sato, Y. and Isono, S. (2017) Mask Ventilation during Induction of General Anesthesia: Influences of Obstructive Sleep Apnea. Anesthesiology, 126, 28-38. https://doi.org/10.1097/ALN.0000000000001407

[8] Safar, P. (1958) Ventilatory Efficacy of Mouth-to-Mouth Artificial Respiration: Airway Obstruction during Manual and Mouth-to-Mouth Artificial Respiration. Journal of the American Medical Association, 167, 335-341.

[9] Safar, P. and Redding, J. (1959) The "Tight Jaw" in Resuscitation. Anesthesiology, 20, 701-702.

[10] Vogel, J.E., Mulliken, J.B. and Kaban, L.B. (1986) Macroglossia: A Review of the Condition and a New Classification. Plastic and Reconstructive Surgery, 78, 715-723. 
[11] Alhajj, M.N., Khalifa, N., Abduo, J., Amran, A.G. and Ismail, I.A. (2017) Determination of Occlusal Vertical Dimension for Complete Dentures Patients: An Updated Review. Journal of Oral Rehabilitation, 44, 896-907.

https://doi.org/10.1111/joor.12522

[12] Racine, S.X., Solis, A., Hamou, N.A., Letoumelin, P., Hepner, D.L., Beloucif, S. and Baillard, C. (2010) Face Mask Ventilation in Edentulous Patients: A Comparison of Mandibular Groove and Lower Lip Placement. Anesthesiology, 112, 1190-1193. https://doi.org/10.1097/ALN.0b013e3181d5dfea

[13] Yildiz, T.S., Solak, M. and Toker, K. (2005) The Incidence and Risk Factors of Difficult Mask Ventilation. Journal of Anesthesia, 19, 7-11. https://doi.org/10.1007/s00540-004-0275-z

[14] Kheterpal, S., Martin, L., Shanks, A.M. and Tremper, K.K. (2009) Prediction and Outcomes of Impossible Mask Ventilation: A Review of 50,000 Anesthetics. Anesthesiology, 110, 891-897. https://doi.org/10.1097/ALN.0b013e31819b5b87

[15] El-Orbany, M. and Woehlck, H.J. (2009) Difficult Mask Ventilation. Anesthesia and Analgesia, 109, 1870-1880. https://doi.org/10.1213/ANE.0b013e3181b5881c

[16] Ikeda, A., Isono, S., Sato, Y., Yogo, H., Sato, J., Ishikawa, T. and Nishino, T. (2012) Effects of Muscle Relaxants on Mask Ventilation in Anesthetized Persons with Normal Upper Airway Anatomy. Anesthesiology, 117, 487-493.

https://doi.org/10.1097/ALN.0b013e3182668670 